\title{
Desarrollo de habilidades sociales básicas y avanzadas en adolescentes y jóvenes con discapacidad visual
}

\author{
Development of basic and advanced social skills in adolescents and young people with \\ visual disabilities \\ Desenvolvimento de habilidades sociais básicas e avançadas em adolescentes e jovens com \\ deficiência visual
}

\section{ARTÍCULO DE INVESTIGACIÓN}

\author{
Mayra Aguilar Verdugo \\ maygabiav@gmail.com \\ https://orcid.org/0000-0002-4305-9630 \\ María Ochoa Guerrero \\ marules85@hotmail.com \\ https://orcid.org/0000-0002-3866-8781 \\ Edinson Rogel Díaz \\ edinsonrogel94@outlook.com \\ https://orcid.org/0000-0002-9632-2236 \\ Graciela Urías Arbolaez \\ gracielauriasarbolaez@yahoo.es \\ https://orcid.org/0000-0001-5927-3351 \\ Universidad Nacional de Educación, Cañar-Ecuador
}

Recibido 08 de enero 2021 | Arbitrado y aceptado 15 de febrero 2021| Publicado en 01 abril 2021

\section{RESUMEN}

El estudio constituye las acciones que tienen lugar en el proceso de enseñanza y aprendizaje de adolescentes y jóvenes con discapacidad visual en Unidad de Educación Especial "Claudio Neira Garzón", de la ciudad de Cuenca-Ecuador. Se fundamentó en el paradigma cualitativo a través del método el estudio de caso grupal, el objeto de estudio fue potenciar las habilidades sociales básicas y avanzadas en el grupo de estudiante que integraron la muestra de la investigación. Las técnicas utilizadas fueron: la observación participante, entrevista semiestructurada y escala de habilidades sociales, con sus respectivos instrumentos. La investigación derivó al diseño de un sistema de actividades dividida en tres fases, siguiendo un orden secuenciado, basadas en estrategias didácticas tales como; trabajo cooperativo y el juego de roles. La propuesta fue evaluada mediante el criterio de especialistas, obteniéndose resultados positivos, debido principalmente a que está contextualizada a la escuela, familia y comunidad, con recursos y estrategias didácticas accesibles considerando potencialidades, intereses y necesidades de los educandos.

Palabras clave: Discapacidad visual; habilidades sociales básicas; habilidades sociales avanzadas; sistema de actividades

\section{ABSTRACT}

This work constitutes an approach to the actions that take place in school classrooms and to the didactic and pedagogical processes. It was based on the qualitative paradigm, the group case study method, the object of study was to enhance basic and advanced social skills in adolescents and young people with visual disabilities of the Special Education Unit "Claudio Neira Garzón". The techniques used were: participant observation, semistructured interview and social skills scale, with their respective instruments. The research led to the design of a system of activities divided into three phases, in sequenced order, based on didactic strategies such as; cooperative work and role play. The proposal was evaluated through the criteria of specialists, obtaining positive results, mainly due to the fact that it is contextualized to the school, family and community, with accessible teaching resources and strategies considering the potential, interests and needs of the students.

Key words: Visual disability; Basic social skills; advanced social skills; activity system

\section{RESUMO}

Este trabalho constitui uma aproximação às ações que acontecem nas salas de aula das escolas e aos processos didático-pedagógicos. A partir do paradigma qualitativo, método de estudo de caso em grupo, o objeto de estudo foi o aprimoramento de habilidades sociais básicas e avançadas em adolescentes e jovens com deficiência visual da Unidade de Educação Especial "Claudio Neira Garzón”. As técnicas utilizadas foram: observação participante, entrevista semiestruturada e escala de habilidades sociais, com seus respectivos instrumentos. A pesquisa levou ao desenho de um sistema de atividades dividido em três fases, em ordem sequencial, a partir de estratégias didáticas como; trabalho cooperativo e dramatização. A proposta foi avaliada através de critérios de especialistas, obtendo resultados positivos, principalmente pelo fato de ser contextualizada para a escola, família e comunidade, com recursos e estratégias pedagógicas acessíveis considerando as potencialidades, interesses e necessidades dos alunos.

Palavras-chave: Habilidades sociais básicas; habilidades sociais avançadas; sistema de atividade; deficiência visual 
INTRODUCCIÓN

En Latinoamérica en el caso de países cercanos tales como Colombia, el Instituto Nacional de Ciegos (INCI), precisa que en 2006 se diagnosticaron, $1100 \quad 000$ personas con afectación visual, distribuidos en $80 \%$ casos con baja visión y $20 \%$ ceguera, el caso es similar en Perú representando un $32 \%$ de la población entre baja visión y ceguera total.

En Ecuador según datos del Consejo Nacional para la Igualdad de Discapacidades (CONADIS, 2020) hay un total de 55.843 personas con discapacidad visual repartidas en la mayoría del territorio nacional, de este número 23.114 corresponde al género femenino, 32.727 al género masculino y 2 al LGTBI.

En la provincia de Azuay las personas con discapacidad visual ascienden a 3.748 , de los cuales 1.785 corresponden al género femenino y 1.963 al género masculino. (CONADIS, 2020).

Es evidente que, las realidades son diferentes, prueba de ello es el alto índice de personas con discapacidad visual en países en vías de desarrollo. Además, contextualizado al Ecuador donde se encuentra el objeto de investigación, demuestra tener un porcentaje similar entre hombres $\mathrm{y}$ mujeres, lo antes expuesto permite reflexionar la prioridad que se debe dar al diseño de proyectos innovadores y accesibles que equiparen oportunidades orientadas a mejorar la calidad de vida de esta población.

Por otra parte, es importante contemplar que todas las personas somos un mundo diverso, con características propias que nos hacen inigualables en varios aspectos, dentro de la investigación no se generalizaron capacidades, habilidades, limitaciones, tengan discapacidad o no, ya que al tener características propias fue necesario conocer el contexto y la realidad que envuelve su desarrollo. Es así, que desde la investigación aplicada en la mencionada institución educativa, se analizaron el aspecto social y la percepción del entorno. Por todo ello, es importante tener en consideración la diferencia existente en el desarrollo de la competencia social en personas normo videntes con relación a personas con discapacidad visual, durante mucho tiempo se ha creído que su fuerte es la comunicación oral y que por ende la parte social es innata, sin considerar que todo depende de un contexto rico o pobre en estimulación, sin embargo, existen autores que plantean diversos conceptos, tales como el que se expone a continuación:

Las habilidades son el resultado
de la interacción de una
persona concreta, con sus
capacidades y limitaciones, con
el contexto social en el que esta
se encuentra, para dar sentido
al aprendizaje y el hecho de
aprender del otro y con el otro.
(Caballo y Verdugo, 2013, p.
32).

Las políticas de educación e inserción laboral en Ecuador de personas con 
discapacidad plantean que es necesario preparar a los adolescentes y jóvenes con discapacidad visual en el desarrollo de las habilidades sociales para adaptarse al campo laboral y social, y con ello satisfacer necesidades $\quad \mathrm{y} \quad$ oportunidades imprescindibles para los seres humanos.

Cabe indicar que se trabajó con el 6to y 7 mo de Básica de la unidad educativa antes mencionada, distribuidos de la siguiente manera:) 6to grado con un total de dos alumnos, un niño y una niña; y $7 \mathrm{mo}$ grado, con un total de tres alumnos, aula unidocente. Los métodos de trabajo dentro del proceso de enseñanza aprendizaje son predominantemente individuales, centrados en el uso único de recursos tales como; computadora, ábaco, regleta $\mathrm{y}$ punzón, los cuales no permiten el aprendizaje colaborativo, la tutoría de apoyo entre iguales afectando la interacción entre los estudiantes y su contexto, en esencia la metodología empleada por el docente tiene un marcado carácter personalizado y se descuida lo grupal, lo colectivo, lo cual no potencia el desarrollo de habilidades sociales en los estudiantes.

El estudio se concibe desde los supuestos teóricos, epistemológicos y metodológicos del Modelo Pedagógico de la Universidad Nacional de Educación (UNAE, s.f.) de Ecuador, específicamente, en el proceso de prácticas preprofesionales desarrolladas en los ciclos superiores (7mo y 9no) de la carrera de Educación Especial, itinerario Discapacidad Sensorial. El proceso investigativo refleja el estudio del contexto escolar, su realidad y diversidad en el desarrollo del proceso de enseñanza y aprendizaje en adolescentes y jóvenes con discapacidad visual del 6to y $7 \mathrm{mo}$ año de educación básica de la Unidad de Educación Especial "Claudio Neira Garzón”.

Para comprender la problemática que se investiga es necesario partir de la idea confirmada por las ciencias de que la mayoría de personas perciben el mundo mediante el sentido de la vista, a través de él se obtiene el $87 \%$ de la información sobre el mundo que nos rodea, nos ayuda a adquirir todas experiencias nuevas, a orientarnos, a relacionarnos, no obstante, cuando este sentido se halla ausente o se encuentra atrofiado por distintas causas, cambia la forma y estilo de percibir $y$ aprender sobre el mundo.

Lo anterior expuesto, se ha convertido en pieza clave para la presente investigación. Conocer e interpretar la realidad dentro de las estimaciones declaradas de los porcentajes en torno a la discapacidad visual, nos ha llevado a indagar en estudios internacionales $y$ nacionales de diferentes organizaciones que se preocupan en proponer políticas y planes de mejora para la población con discapacidad visual, en el marco del respeto y equidad de oportunidades. En este sentido importantes organismos tales como; el Instituto Nacional de Ciegos y el Consejo Nacional para la Igualdad de Discapacidades, son considerados en la investigación como referentes legales para 
crear conciencia y establecer la normativa de que, a pesar de ser un grupo minoritario, son parte de la sociedad.

Las problemáticas antes expuestas, que son resultados de los datos empíricos obtenidos en la investigación, condujeron a la siguiente pregunta científica:

¿Cómo potenciar las habilidades sociales básicas y avanzadas en adolescentes y jóvenes con discapacidad visual del 6to y $7 \mathrm{mo}$ de básica de la Unidad de Educación Especial "Claudio Neira Garzón"?

\section{MÉTODO}

El marco metodológico estuvo estructurado acorde a las necesidades del proceso de investigación ligada a la realidad del contexto educativo estudiado. Se consideró el proceso de investigación cualitativa, que es considerado "como estudios sobre el quehacer cotidiano de las personas o de grupos pequeños. En este tipo de investigación interesa lo que la gente dice, piensa, siente o hace; sus patrones culturales; el proceso y el significado de sus relaciones interpersonales y con el medio". (Gonzáles, 2009, p. 71).

Por otra parte, en relación al planteamiento del problema inicial ¿Cómo potenciar las habilidades sociales básicas y avanzadas en adolescentes y jóvenes con discapacidad visual del 6to y $7 \mathrm{mo}$ de básica de la Unidad de Educación Especial “Claudio Neira Garzón"? Para poder comprender dicha realidad se partió del paradigma cualitativo, con una mirada centrada en la realidad del objeto de estudio y el mutuo acuerdo, de tal manera que las dos partes se complementan en el proceso, para que esto sea posible se trabajó desde el método estudio de caso grupal.

Las técnicas de investigación fueron la entrevista semiestructurada, observación participante y la escala de habilidades sociales y como instrumentos: el cuestionario de entrevista, guías de observación, diarios de campo y cuestionario de habilidades sociales. Finalmente, la evaluación de la propuesta innovadora planteada desde la validación del criterio de especialistas.

\section{RESULTADOS Y DISCUSIÓN}

\section{Criterio de especialistas, resultados}

A continuación, se muestran los resultados de la validación de la propuesta mediante el criterio de especialistas, obteniéndose los siguientes resultados:

\section{Análisis e interpretación del criterio "claridad",}

La dimensión 1. La redacción de la propuesta usa lenguaje comprensible para otros actores de la comunidad educativa y la familia interesados en su réplica.

Se obtuvo un $57.1 \%$ correspondiente a la escala totalmente y con un $42.9 \%$ a mucho. Lo que implicó que las observaciones de los especialistas concordaron que la propuesta tiene un lenguaje compresible $y$ de fácil entendimiento para quienes vayan a ser uso de esta. 
Claridad: La propuesta es fácil de entender y, por ello, podria ser aplicada por docentes o familias sin mayor dificultad

1. La redacción de la propuesta usa lenguaje comprensible para otros actores de la comunidad educativa y la familia interesados en su réplica.

7 respuestas

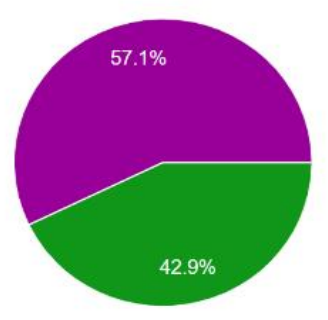

Nada

Poco

Medianamente

- Mucho

- Totalmente

Gráfico 1. Tabulación primer indicador del criterio "claridad. (Fuente: Aguilar \& Rogel, 2021).

Segunda dimensión: La escritura de la propuesta considera las reglas ortográficas del idioma.

Se obtiene un $71.4 \%$ que corresponde a la escala totalmente, $14.3 \%$ mucho y $14.3 \%$ en la escala medianamente. La interpretación de estos datos induce a que, a pesar de tener un resultado máximo en este indicador, es necesario hacer los ajustes en cuanto a las reglas ortográficas del idioma.

2. La escritura de la propuesta considera las reglas ortográficas del idioma.

7 respuestas

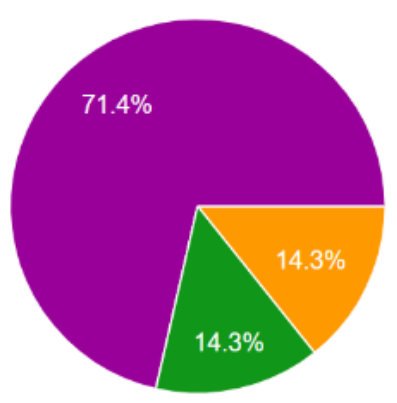

Totalmente

Gráfico 2. Tabulación segundo indicador del criterio "claridad". (Fuente: Aguilar \& Rogel, 2021).

Tercera dimensión. La estructura gramatical es correcta y guarda concordancia con las reglas del idioma español. 
Se obtuvo un $71.4 \%$ en la escala totalmente, $14.3 \%$ mucho y $14.3 \%$ medianamente. Lo que nos lleva a interpretar nuevamente que se ha conseguido un alto porcentaje de valoración de acuerdo a las reglas del idioma establecidas en el desarrollo de la propuesta, no obstante, un porcentaje mínimo considera hacer correcciones en ciertos apartados del sistema de actividades.
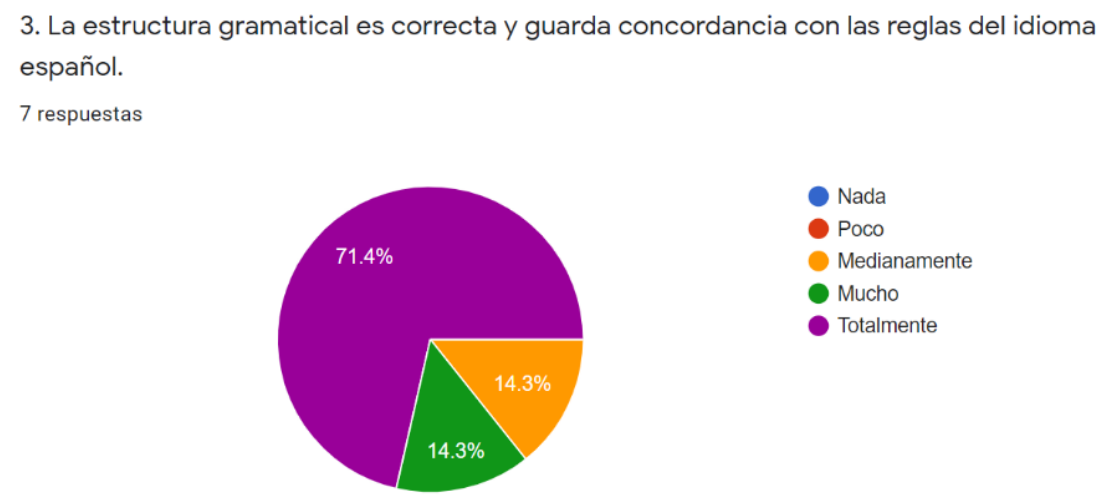

Gráfico 3. Tabulación tercer indicador del criterio "claridad". (Fuente: Aguilar \& Rogel, 2021).

La cuarta dimensión. El significado de las palabras y oraciones que se plantean en la propuesta es el correcto (estructura semántica de la lengua).

Con relación al criterio claridad se obtuvo nuevamente un alto porcentaje de $57.1 \%$ que equivalía a la escala totalmente y un $42.9 \%$ correspondiente a mucho. La interpretación del mismo permitió evidenciar que la propuesta tiene un nivel de estructura semántica acorde los contextos en los cuales se pretende trabajar.

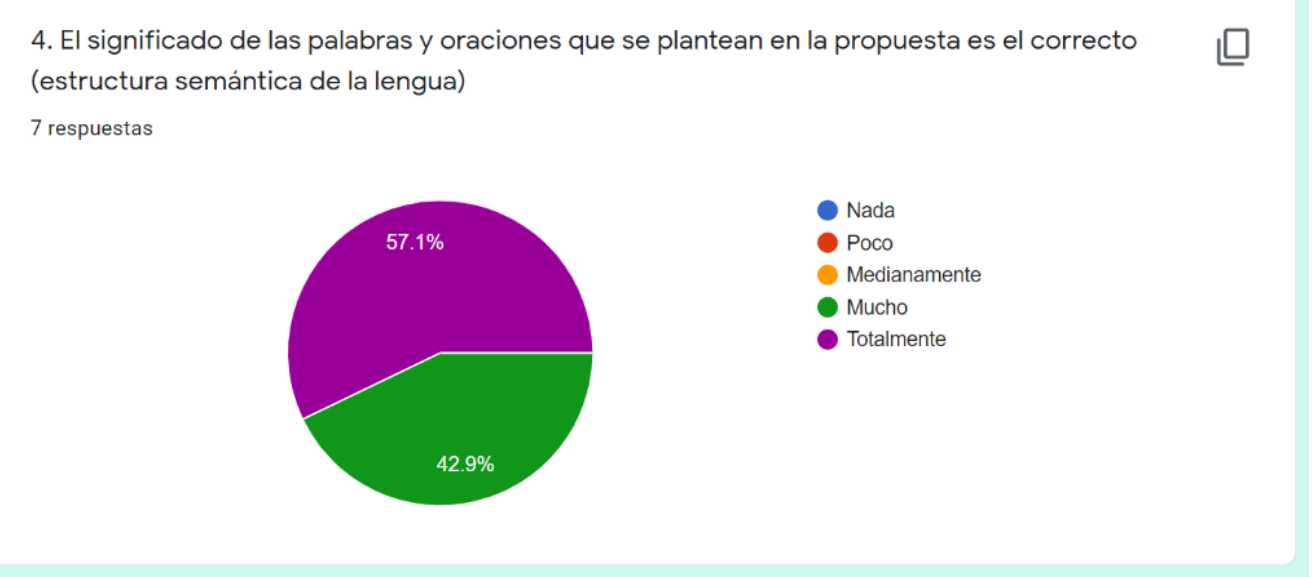

Gráfico 4. Tabulación cuarto indicador del criterio "claridad. (Fuente: Aguilar \& Rogel, 2021). 
Mayra Aguilar Verdugo; Edinson Rogel Díaz; María Ochoa Guerrero, y Graciela Vrías Arbolaez

Observaciones de la validación de especialistas de acuerdo al criterio "claridad".

- Aquel que revise la intervención está en capacidad de construir significados en base a lo expuesto, el entendimiento está garantizado, lo cual la presente propuesta cumple.

- La propuesta es muy clara y se entiende fácilmente. Es necesario utilizar un lenguaje factible y accesible para que todos los miembros de la comunidad educativa puedan comprender y aplicar de manera sencilla las actividades planteadas. La propuesta cumple con estos aspectos.

- No se hace ninguna observación en este criterio.

- Crear una guía breve para la búsqueda de actividades según el interés del lector.

- La propuesta es clara brinda una guía sencilla y entendible de las actividades para mejorar las habilidades sociales, las cuales pueden ser fácilmente adaptadas al entorno al cual se va a trabajar y con materiales disponibles en escuela y hogar.

\section{Análisis e interpretación del criterio "pertinencia"}

Se presentan de forma detallada el análisis e interpretación del segundo criterio "pertinencia" por cada dimensión.

Primera dimensión. Los antecedentes y justificación de la propuesta evidencian su importancia con relación al tema que aborda.

Se obtuvo un $85.7 \%$ correspondiente a la escala totalmente y un $14.3 \%$ en la escala mucho. Se comprobó una vez más en la validación de especialistas un mutuo acuerdo en la idea de que la propuesta cumplía con una justificación que respalda la importancia del sistema de actividades para potenciar las habilidades sociales en jóvenes y adolescentes con discapacidad visual.

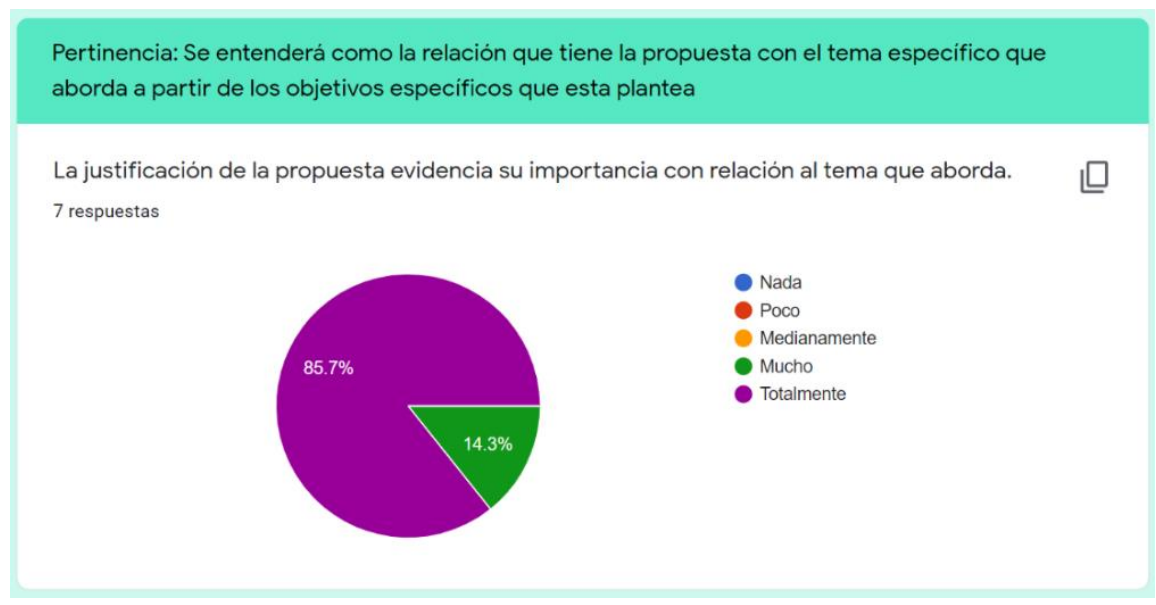

Gráfico 5. Tabulación primer indicador del criterio "pertinencia". (Fuente: Aguilar \& Rogel, 2021). 
Mayra Aguilar Verdugo; Edinson Rogel Díaz; María Ochoa Guerrero, y Graciela Vrías Arbolaez

Segunda dimensión. Los objetivos generales y específicos se relacionan con la temática que aborda la propuesta.

Se obtuvo un $85.7 \%$ en la escala totalmente y un $14.3 \%$ en la escala mucho. Se dedujo un alto porcentaje en la idea de que la temática abordada en la propuesta cumplía con el objetivo general y los específicos.

Los objetivos general y especifico se relacionan con la temática que aborda la propuesta.

7 respuestas

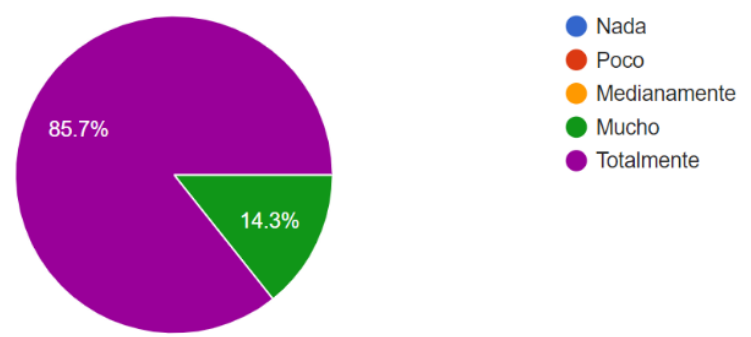

Gráfico 6. Tabulación segundo indicador del criterio "pertinencia". (Fuente: Aguilar \& Rogel, 2021).

Tercera dimensión. La categoría general que se pretendió potenciar se desglosa en dimensiones e indicadores más particulares. Representa el $71.4 \%$ en la escala totalmente y $28.6 \%$ en la escala mucho. Por ende, se cumplió de forma clara con las dimensiones e indicadores que orientan la propuesta.
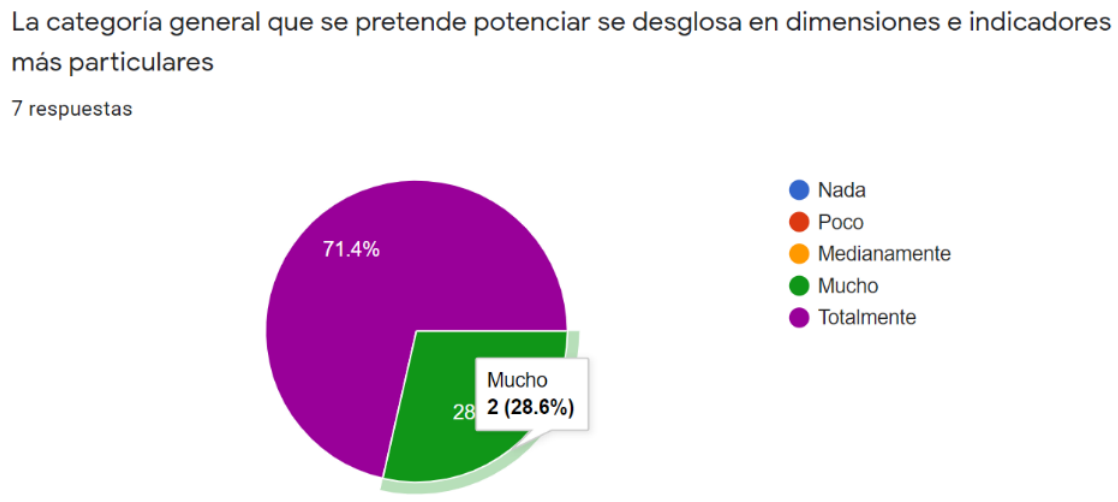

Gráfico 7. Tabulación tercer indicador del criterio "pertinencia". (Fuente: Aguilar \& Rogel, 2021). 
Cuarta dimensión. Los bloques de actividades (circuitos) tienen relación con las dimensiones e indicadores que se pretenden potenciar.

Se logra $85.7 \%$ en la escala totalmente y $14.3 \%$ en la escala mucho. Determinó que el sistema de actividades cumplía con cada una de las habilidades sociales que se va a potenciar en jóvenes y adolescentes con discapacidad visual.

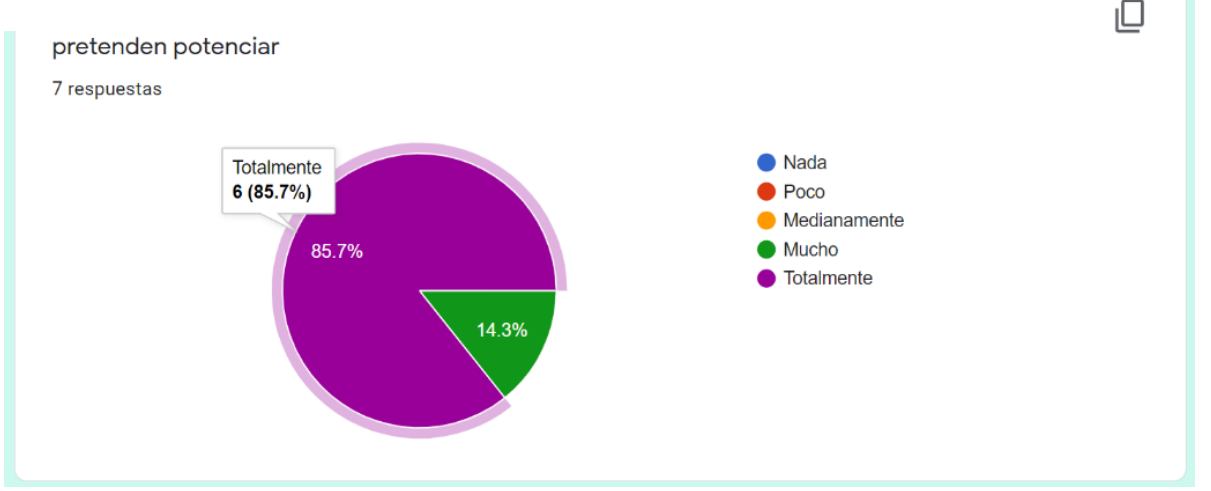

Gráfico 8. Tabulación cuarto indicador del criterio "pertinencia”. (Fuente: Aguilar \& Rogel, 2021).

\section{Análisis e interpretación del criterio "coherencia"}

A continuación, se presentan de forma detallada el análisis e interpretación del tercer criterio "coherencia" para cada dimensión.

Primera dimensión. La Fase 1, establece con precisión el sistema de actividades sobre los que busca potenciar y la estrategia didáctica que permiten alcanzar ese propósito.

Un $85.7 \%$ corresponde a la escala totalmente y $14.3 \%$ a la escala mucho. Dichos resultados afirmaron que la esta propuesta cumple con una relación lógica y directa entre el sistema de actividades y habilidades sociales a potenciar.

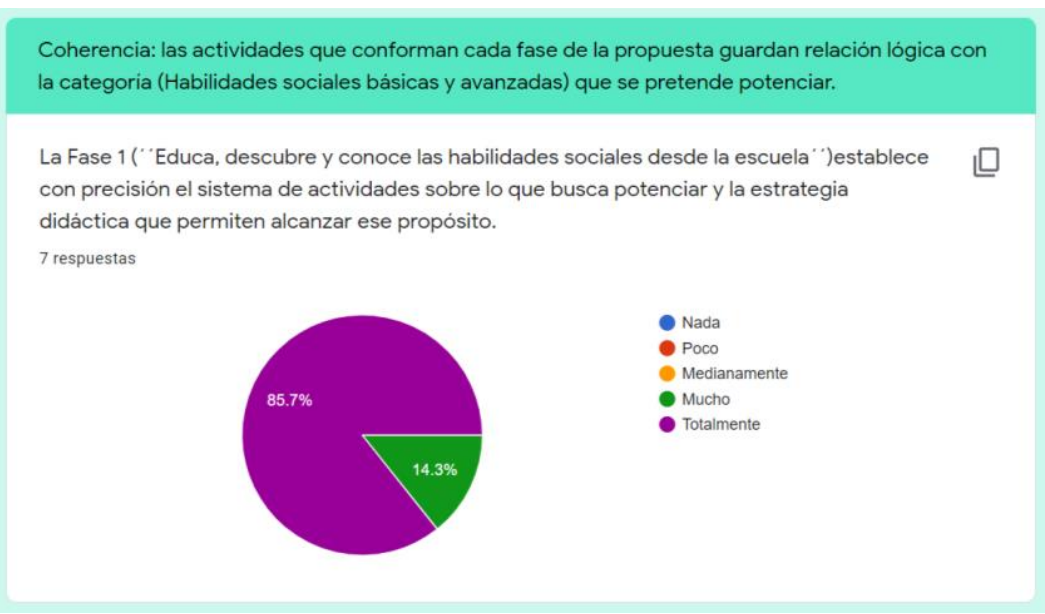

Gráfico 9. Tabulación primer indicador del criterio "coherencia”. (Fuente: Aguilar \& Rogel, 2021). 
Mayra Aguilar Verdugo; Edinson Rogel Díaz; María Ochoa Guerrero, y Graciela Vrías Arbolaez

La segunda dimensión. La Fase 2, establecer el logro obtenido como establece con precisión el sistema de actividades sobre los que busca potenciar y la estrategia didáctica que permiten alcanzar ese propósito.

Presenta un $85.7 \%$ que corresponde a la escala totalmente y un $14.3 \%$ a la escala mucho. Dichos resultados permitieron investigadores, entorno a la precisión existente entre el diseño del sistema de actividades y las estrategias didácticas utilizadas para potenciar habilidades sociales básicas y avanzadas en adolescentes y jóvenes con discapacidad visual.

\footnotetext{
La Fase 2 ( 'Educa, descubre y conoce las habilidades sociales desde la familia")establece con precisión el sistema de actividades sobre lo que busca potenciar y la estrategia didáctica que permiten alcanzar ese propósito.

7 respuestas
}

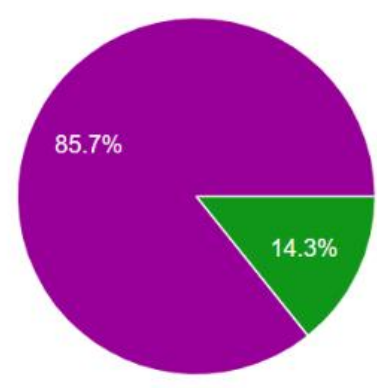

Gráfico 10. Tabulación segundo indicador del criterio "coherencia". (Fuente: Aguilar \& Rogel, 2021)

Tercera dimensión. La Fase 3, los indicadores del criterio "coherencia" establece con precisión el sistema de actividades sobre los que busca potenciar y la estrategia didáctica que permiten alcanzar ese propósito.

Establece $\quad 87.7 \%$ en la escala totalmente y un $14.3 \%$ en la escala mucho. En es importante mencionar que los resultados obtenidos en cada uno de desde la fase uno, dos y tres concordaron que se cumple con la valoración máxima de acuerdo al diseño del sistema de actividades bajo estrategias didácticas que permiten potenciar las habilidades sociales básicas y avanzadas en un grupo de estudiantes con discapacidad visual. 


\footnotetext{
La Fase 3 (Escuela, familia y contexto entorno de desarrollo social)establece con precisión el sistema de actividades sobre lo que busca potenciar y la estrategia didáctica que permiten alcanzar ese propósito.

7 respuestas
}
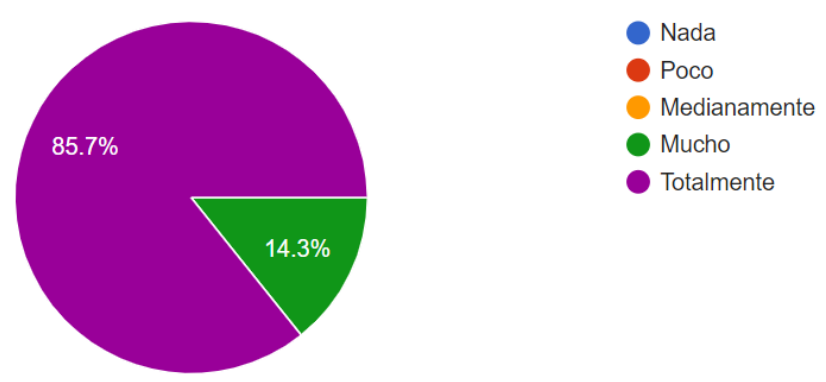

Gráfico 11. Tabulación tercer indicador del criterio "coherencia". Fuente: Aguilar \& Rogel, (2021).

Es importante mencionar que los resultados obtenidos en cada uno de los indicadores del criterio "coherencia" cumplieron con la valoración máxima de acuerdo al diseño del sistema de actividades bajo estrategias didácticas que permiten potenciar las habilidades sociales básicas y avanzadas en el grupo de estudiantes con discapacidad visual. Además, permite concluir que la propuesta puede ser modificada a otros contextos considerando las necesidades, e intereses, etc.

\section{Análisis e interpretación del criterio "relevancia"}

Primera dimensión. El sistema de actividades propuestas para la Fase 1, son importantes en el propósito de alcanzar los objetivos establecidos.

Se obtiene un $85.7 \%$ que corresponde a la escala totalmente y un $14.3 \%$ a la escala mucho. Al obtener un porcentaje alto en esta dimensión se evidenció que efectivamente el sistema de actividades planteados en la fase uno, cumplen con los objetivos establecidos. 


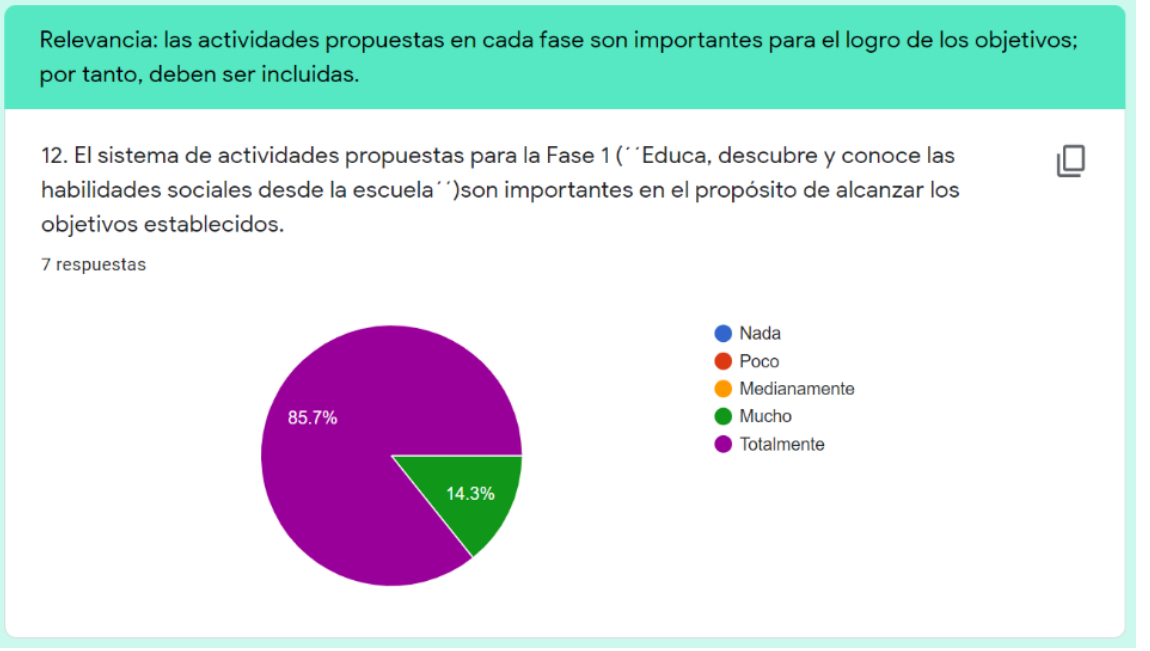

Gráfico 12. Tabulación primer indicador del criterio "relevancia". Fuente: Aguilar \& Rogel, (2021).

Segunda dimensión. El sistema de actividades para la Fase 2, son importantes en el propósito de alcanzar los objetivos establecidos.

Se obtiene un $85.7 \%$ que corresponde a la escala totalmente y un $14.3 \%$ a la escala mucho. Dichos resultados comprobaron que el sistema de actividades planteadas en la fase dos, cumple con los objetivos establecidos.
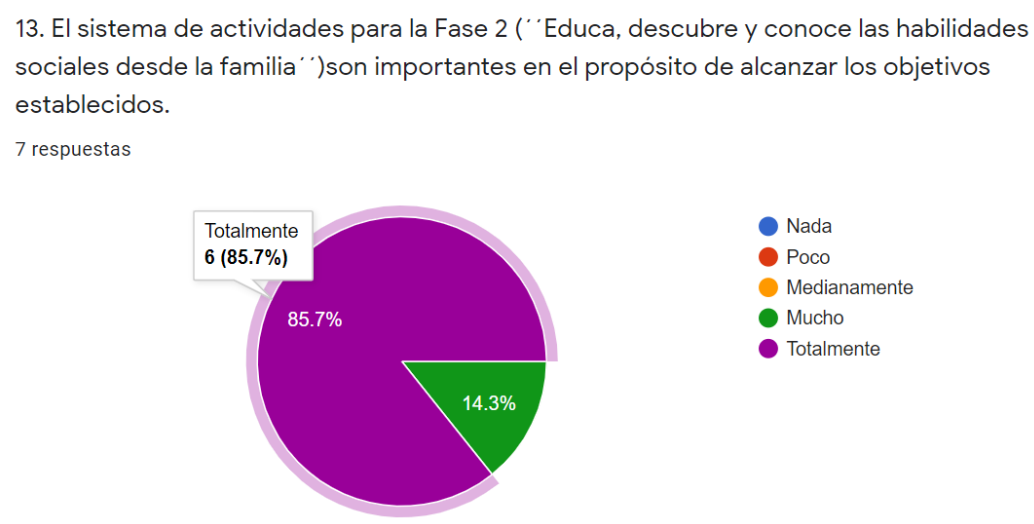

Gráfico 13. Tabulación segundo indicador del criterio "relevancia". (Fuente: Aguilar \& Rogel, 2021).

Tercera dimensión. El sistema de actividades propuestas para la Fase 3, son importantes en el propósito de alcanzar los objetivos establecidos.

Un $85.7 \%$ que corresponde a la escala totalmente y un $14.3 \%$ a la escala mucho. Al igual que en la dimensión uno y dos del criterio relevancia, se obtiene un alto grado de calificación, debido a que cumple con un sistema de actividades en concordancia a los objetivos establecidos en cada fase de la propuesta. 


\begin{abstract}
14. El sistema de actividades propuestas para la Fase 3 (Escuela, familia y contexto entorno de desarrollo social)son importantes en el propósito de alcanzar los objetivos establecidos.

7 respuestas
\end{abstract}

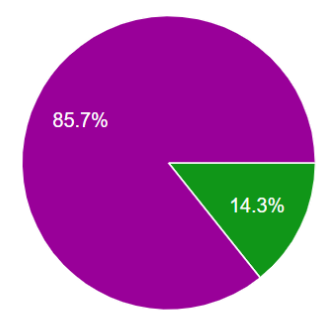

Nada

Poco

Medianamente

Mucho

Totalmente

Gráfico 14. Tabulación tercer indicador del criterio "relevancia". (Fuente: Aguilar \& Rogel, 2021).

La propuesta fue de gran impacto para cada uno de los especialistas en relación a los criterios que se estableció para su evaluación, se reitera en cada una de las opiniones dadas que, el sistema de actividades responde a los objetivos planteados, a las necesidades, características, estilos e intereses de los estudiantes, por otro lado, se resaltó que el diseño de la propuesta tiene una visión contextualizada a la familia y comunidad para potenciar las habilidades sociales básicas y avanzadas.

Se consideró que las actividades pueden ser realizadas por otros jóvenes y adolescentes que presentan discapacidad visual en diferentes instituciones del país en dependencia del nivel de desarrollo de las habilidades sociales que presentan, el contexto donde viven, las características de la familia y de los intereses de estos.

\section{Análisis final de los resultados}

La metodología de enseñanzaaprendizaje responde a procesos conceptuales y procedimentales, así como conjuntos de actividades interdependientes y consecutivas guiadas por el docente.

Los estudiantes manifiestan preferir la escuela a su hogar debido a que en éste último no salen a lugares públicos.

Los contenidos se basan en el dictado, repetición, domina la clase magistral, trata en lo posible de relacionar lo enseñado con actividades del diario vivir.

El docente establece diálogos relacionados a la discapacidad, y enfatiza que esta no es un limitante para lograr metas.

Usa recursos diseñados para estudiantes con discapacidad visual como el ábaco para operaciones matemáticas, enseña técnicas de resolución de operaciones ideadas por el docente para mejorar su transición de educación especial a regular.

Los recursos se utilizan de forma reiterada en las asignaturas.

Orientación y movilidad del grupo es acertada en alumnos con baja visión y trabajo colaborativo entre compañeros del aula. 
Existen intereses comunes en el grupo, es el caso de los videojuegos, el tema de conversación de los alumnos en los espacios de ocio.

Los estudiantes expresan sus intereses mediante conversaciones espontáneas que surgen de la interacción con el grupo, mostraron gran interés en la idea de crear productos de aseo personal y hacer una microempresa.

Lo que caracteriza a este grupo es la apertura en cuanto a compartir su conocimiento entre ellos, si alguno del grupo no sabe algo inmediatamente otro compañero se lo explica.

Se logró acompañar los estudiantes por los distintos equipos multidisciplinarios con el propósito de observar su interacción con personas diferentes a su aula, como es actitud frente a las actividades que realizan con diferentes profesionales y la reacción a la misma.

En escenarios diferentes al aula, los estudiantes cambian su comportamiento reduciendo limitándose a escuchar $\mathrm{y}$ obedecer, en terapia ocupacional muestran mayor interés y disposición en las actividades a realizar.

La información obtenida en las guías de observación basada en indicadores sobre las habilidades sociales, aplicada a cada estudiante, destaca en los estudiantes tal es el caso de las habilidades sociales básicas y avanzadas.

La capacidad de escuchar cuando alguien habla, es difícil mantenerlo pues entre compañeros susurran lo que trae consigo distorsión de información por parte del docente y llamado de atención constantemente.

Iniciar una conversación con otras personas ajenas al grupo clase, se vuelve difícil, por ende, prefieren alejarse $\mathrm{y}$ quedarse callados.

El mismo hecho de no escuchar mientras otro habla, le dificulta seguir instrucciones por mínima que sea, por tanto, se repite con frecuencia.

\section{Pautas para la creación del sistema de actividades}

En consecuencia, de acuerdo a lo mencionado en los párrafos anteriores, se determina con claridad el plan de mejora dirigido a potenciar habilidades sociales básicas $\mathrm{y}$ avanzadas en adolescentes $\mathrm{y}$ jóvenes con discapacidad visual, desde estrategias didácticas participativas, que rompa el enfoque de clase magistral, el uso de recursos únicos, centrado en el docente y el entorno escolar, todo ello desde la base del respeto a los ritmos, necesidades $y$ estilos.

Dentro de la primera fase se trabaja en el ambiente escolar considerando habilidades sociales básicas y avanzadas. El diseño del sistema de actividades planteadas permite interactuar dentro del contexto áulico, así como con la comunidad educativa. Cada actividad va en función de una habilidad social y un nombre acorde a la habilidad, caracterizado por ser accesible, novedoso, como se muestra a continuación. 


\section{FASE I}

"Educa, descubre y conoce las habilidades sociales desde la escuela"

\section{HABILIDADES SOCIALES BÁSICAS}

1. Escuchar.

2. Iniciar una conversación.

3. Mantener una conversación.

4. Presentarse.

5. Presentar a otras personas.

6. Hacer un cumplido.

\section{HABILIDADES SOCIALES AVANZADAS}

7. Seguir Instrucciones.

8. Convencer a los demás. Creatividad -Video Game".

9. Participar.

10. Dar instrucciones.

\section{SISTEMA DE ACTIVIDADES}

Actividad 1: "El jefe del Centro Comercial". Actividad 2: "Conociéndonos aún más versión compañeros". Actividad 3: "El teléfono descompuesto".

Actividad 4: "Hola, Me presento".

Actividad 5: "Te presento a".

Actividad 6: "Cumplidos para todos".

\section{SISTEMA DE ACTIVIDADES}

Actividad 7: "Sígueme el paso".

Actividad 8: "Mi post publicitario", "Convenciendo desde mi

Actividad 9: "Opino y participo".

Actividad 10: "Encontrando pistas".
En la fase dos, las actividades van orientadas al ambiente familiar, es decir el protagonismo de estas recaen en la familia como punto fuerte para el desarrollo de habilidades sociales. Cada actividad fue creada en función de la habilidad social a potenciar y están pensadas para ser aplicadas de forma secuenciada. Las actividades son dinámicas y creadas para motivar la participación de los miembros de la familia de igual manera se hace uso de recursos accesible que facilitan su aplicación. A continuación, se muestran las habilidades sociales a potenciar con sus respectivas actividades. 
Mayra Aguilar Verdugo; Edinson Rogel Díaz; María Ochoa Guerrero, y Graciela Vrías Arbolaez

\section{FASE II}

"Educa, descubre y conoce las habilidades sociales desde la familia"

\section{HABILIDADES SOCIALES BÁSICAS}

1) Escuchar.

2) Iniciar una conversación.

3) Mantener una conversación. versión familia"

4) Formular una pregunta.

5) Dar las gracias.

6) Presentarse.

7) Presentar a otras personas.

8) Hacer un cumplido.

HABILIDADES SOCIALES AVANZADAS

9) Participar.

10) Dar instrucciones.

11) Seguir Instrucciones.

12) Convencer a los demás. creatividad video-Game- Versión Familia". Familia".

\section{SISTEMA DE ACTIVIDADES}

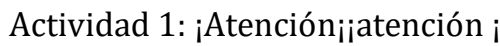

Actividad 2: "Cadena de Nombres"

Actividad 3: "Caja Mágica” "Conociéndote aún más,

Actividad 4: "E buzón de las preguntas"

Actividad 5: "A dar las gracias se ha dicho" Actividad 6: "Visita sorpresa".

Actividad 7: "Conoce a mi familia".

Actividad 8: "La casa de los cumplidos".

\section{SISTEMA DE ACTIVIDADES}

Actividad 9: "Inesperadas vacaciones".

Actividad 10: "Logrémoslo Juntos".

Actividad 11: "Sígueme el paso" versión familia. Actividad 12: Mi post publicitario "convenciendo desde mi

La tercera fase integra lo aprendido en las fases anteriores, esta presenta solamente dos actividades que están dirigidas al contexto social y que abarca tanto el grupo de habilidades sociales básicas como avanzadas.

\section{FASE III}

\section{"Escuela, familia y contexto entorno de desarrollo social"}

\section{HABILIDADES SOCIALES BÁSICAS Y AVANZADAS}

Se trabajan todas las habilidades vistas anteriormente tanto básicas como avanzadas.

\section{SISTEMAS DE ACTIVIDADES}

Actividad 1: "Tú voz, mi radio"

Actividad 2: Stan "las delicias del cacao" "difundo mi producto"

\section{DISCUSIÓN}

La forma en la que la mayoría de las personas perciben el mundo es a través del sentido de la vista, esto nos ayuda a adquirir experiencias nuevas, sin embargo, cuando este sentido se halla ausente o se encuentra atrofiado por distintas causas, cambia la forma de percibir al mundo. La discapacidad visual es la limitación del sentido de la vista, de acuerdo con la Organización Mundial de la Salud (2011), en el mundo existen 253 millones de 
personas con esta condición; de las cuales 227 millones poseen discapacidad visual entre moderada y grave 36 millones presentan ceguera total.

Por otro lado, la Organización Nacional de Ciegos Españoles, ONCE, menciona "la discapacidad visual en general implica la ceguera o deficiencia visual, ambas condiciones caracterizadas por una limitación total o muy seria de la función visual, ocasionada por patologías congénitas, accidentes de cualquier tipo o provocados por virus de diferentes orígenes" (Tobar, 2015, p. 226).

Otra concepción acerca de discapacidad visual está dada por Sevilla (2012) afirma que: "la discapacidad visual es la restricción o ausencia de la capacidad, debida a una deficiencia visual, para llevar a cabo las actividades de la vida cotidiana" (p. 294).

La concepción sobre discapacidad abarcada por Parra y Rojas (2013) mantienen la postura de establecer que discapacidad visual es "un estado de limitación o de menor eficiencia, debido a la interacción entre factores individuales (entre los que se encuentra la deficiencia visual) $\mathrm{y}$ los de un contexto menos accesible" (p. 63).

El aporte establecido por cada autor encamina la investigación en aclarar que existen muchas posturas en cuanto al concepto o definición, pero a su vez permite comprender la similitud entre ellos, tales como la discapacidad visual vista como una dificultad o deficiencia en la función visual que repercute en el desenvolvimiento cotidiano.
Del mismo modo el análisis de información de los resultados de la entrevista aplicada en la Unidad de Educación Especial "Claudio Neira Garzón" dirigida a tres niveles: administrativo, docente de aula y estudiantes del 6to y 7 mo de educación básica de discapacidad visual, de igual modo fue importante contrastar ideas a partir del análisis integrador de un total de 7 diarios de campo, en estos se hacen observaciones de acuerdo a aspectos como: actividades aplicadas por el docente, metodología empleada, intereses, necesidades y ritmo de aprendizaje de los estudiantes, recursos o estrategias educativas aplicadas y observaciones en relación al aprendizaje y participación del grupo o de cada estudiante dentro de la clase o en terapia.

\section{CONCLUSIÓN}

Para finalizar se logró caracterizar el contexto de los adolescentes y jóvenes con discapacidad visual se identificaron algunos intereses tales como: dibujo en estudiantes con baja visión, juegos con características de movimiento y sonido, potencialidades: apoyo grupal a pesar del trabajo individualizado, necesidades en cuanto a espacios de exploración y experimentación de diferentes entornos como medio de aprendizaje e interacción con otros, lo cual permitió constatar las dificultades en el desarrollo de habilidades sociales en correspondencia con la edad cronológica del grupo de estudiantes, estos aspectos permitieron brindar un soporte y funcionalidad en el diseño de la propuesta realizada. 
A partir del diagnóstico se diseñó un sistema de actividades bajo dos estrategias didácticas; juego de roles y trabajo cooperativo. Dicho sistema está orientado al ambiente escolar, familiar y comunitario, cada uno contiene el desarrollo sistemático de habilidades sociales básicas y avanzadas, donde las funciones y responsabilidades cambian en cada contexto. Por todo ello, se considera que la propuesta constituye un recurso efectivo y viable para trabajar el desarrollo y la potenciación de habilidades sociales en educandos con discapacidad visual, puesto que presenta en cada fase una serie de actividades interactivas $y$ estructuradas.

Para fortalecer la viabilidad de la propuesta fue necesario el criterio de especialistas en consideración de la experiencia y conocimiento sobre la discapacidad visual. Los principales resultados obtenidos fueron que el $80 \%$ de la valoración total en concordancia a los 4 criterios establecidos (claridad, pertinencia, coherencia, relevancia) se cumplen satisfactoriamente. Lo cual determinó la eficacia y viabilidad que podrá alcanzar la propuesta.

\section{REFERENCIAS BIBLIOGRÁFICAS}

Caballo E., y Verdugo., A. (2013). Habilidades sociales. Programa para mejorar las relaciones sociales entre niños y jóvenes con deficiencia visual y sus iguales sin discapacidad. Madrid, España: Editorial Artes Gráficas Palermo, S.
CONADIS. (2020). Estadísticas de Discapacidad. https://www.consejodiscapacidades.go b.ec/estadisticas-de-discapacidad/

Gonzáles, H. (2009). Metodología de la Investigación Propuesta, anteproyecto y proyecto. Bogotá, D.C: Eco Ediciones Ltda

Instituto Nacional de Ciegos (INCI) (2006). Estadísticas de afectaciones visuales. Disponible en http://www.inci.gov.co/

Organización Mundial de la Salud - OMS. (2011). Informe Mundial sobre la Discapacidad. Ginebra, Suiza: Ediciones de la OMS. Pág. 3

Parra, D., y Rojas, M. (2013). Necesidades Específicas de Apoyo Educativo del alumnado con discapacidades sensorial y motora. SUMMA PSICOLÓGICA UST. 10(2), 57-72.

Sevilla, M. (2012). Las TIC y la discapacidad visual. En Aplicación de las Tecnologías de la Información y las Comunicaciones en la vida diaria de las personas con discapacidad (pp. 293308). Servizo de Publicacións.

Tobar, K. (2015). Aproximación a las representaciones sociales de profesores universitarios sobre discapacidad visual. Revista de investigación, 39(86), 221-235. Recuperado de http://ve.scielo.org/scielo.php?script=s ci_arttext\&pid=S101029142015000300011\&lng=es\&tlng=pt. UNAE. (s.f). Modelo Pedagógico de la Universidad Nacional del Educación UNAE. Rector. Disponible en: https://unae.edu.ec/wpcontent/uploads/2019/11/modelopedagogico-unae.pdf 
